\title{
ANSIEDADE AO TRATAMENTO ODONTOLÓGICO: PERFIL DE UM GRUPO DE ADULTOS EM SITUAÇÃO NÃO CLÍNICA
}

\author{
Mayra Louise Raiser Santana de Oliveira ${ }^{1}$ \\ Silvana Marchiori Araújo ${ }^{2}$ \\ Elisabete Rabaldo Bottan ${ }^{3}$
}

OLIVEIRA, M. L. R. S. de; ARAÚJO, S. M.; BOTTAN, E. R. Ansiedade ao tratamento odontológico: perfil de um grupo de adultos em situação não clínica. Arq. Cienc. Saúde UNIPAR, Umuarama, v. 19, n. 3, p, 165-170, set./dez. 2015.

\begin{abstract}
RESUMO: O objetivo da pesquisa foi caracterizar um grupo de adultos, em situação não clínica, quanto à ansiedade ao tratamento odontológico. A pesquisa descritiva, do tipo transversal, ocorreu mediante levantamento de dados primários. A população-alvo foi constituída por sujeitos adultos que se encontravam em espaços públicos, no perímetro central de uma cidade de Santa Catarina (Brasil). O plano amostral foi não probabilístico e a obtenção da amostra deu-se por conveniência. O instrumento de coleta de dados foi um questionário elaborado com base na Dental Anxiety Scale (DAS). A amostra foi composta por 304 pessoas ( $56 \%$ do gênero feminino e $44 \%$ do masculino). As idades variaram de 18 a 65 anos. O percentual de mulheres com ansiedade foi de $78 \%$ e o de homens foi $58 \%$. A maioria (62,1\%) dos sujeitos sem ansiedade tinha entre 18 a 34 anos. A consulta em menores intervalos de tempo foi citada por 75,3\%; os procedimentos curativos foram os mais citados $(57,4 \%)$ como motivo da última consulta. Os fatores desencadeadores de ansiedade foram os instrumentos relacionados às intervenções curativas. O maior intervalo de tempo para efetivação da consulta e a consulta em decorrência de dor foram mais frequentes no grau exacerbado. Concluiu-se que a amostra se classificou como portadora de baixo grau de ansiedade; as mulheres demonstraram ser mais ansiosas do que os homens; a maioria do grupo sem ansiedade estava entre os mais jovens; e a frequência da consulta preventiva decresceu à medida que aumentava o grau de ansiedade.
\end{abstract}

PALAVRAS-CHAVE: Ansiedade ao Tratamento Odontológico; Relações Dentista-Paciente; Saúde Bucal; Adulto.

\section{DENTAL ANXIETY: PROFILE OF AN ADULT GROUP IN A NON-CLINICAL SITUATION}

\begin{abstract}
The objective of this research was to characterize a group of adults in a non-clinical situation in terms of their level of anxiety during dental treatment. This is a descriptive, cross-sectional study using a survey of primary data. The target population consists of adult subjects, encountered in public spaces at the downtown area of a town in the State of Santa Catarina (Brazil). It used a non-probability sampling collected by convenience. Data were collected using a questionnaire based on the Dental Anxiety Scale (DAS). The sample consisted of 305 subjects ( $56 \%$ female and $44 \%$ male). Ages ranged from 18 to 65 years. The percentage of women with anxiety was $78 \%$ and of men, $58 \%$. Most of the subjects (62.1\%) who revealed no anxiety were aged between 18 and 34 years. Shorter intervals between visits to the dental clinic were mentioned by $75.3 \%$ of subjects; treatment procedures were the reasons most frequently mentioned for the last visit to the dental clinic. The factor that most triggered anxiety was the equipment used in the treatment. A longer interval between the last visit and the next one due to pain was the most frequently reason mentioned as a cause of exacerbated anxiety level. It was concluded that the sample was classified as having low anxiety; women showed more anxiety than men; most of the subjects in the group who did not suffer anxiety were younger; and the lower the frequency of preventative visits, the higher the level of anxiety.

KEYWORDS: Dental Anxiety; Dentist-Patient Relations; Oral Health; Adult.
\end{abstract}

\section{Introdução}

A ansiedade é uma resposta do ser humano quando exposto a alguma situação de perigo real ou imaginário; trata-se de um estado emocional que faz parte das experiências de vida dos seres humanos, apresentando componentes psicológicos e fisiológicos. Ela não ocorre, exclusivamente, em situações relacionadas ao tratamento odontológico; também, é comum em outras áreas da saúde em geral.

Quando relacionada à Odontologia, a ansiedade pode ser atribuída às grandes mutilações que ocorriam na antiguidade, quando os barbeiros arrancavam os dentes de uma forma dolorosa e sem nenhuma técnica. Hoje em dia, apesar de todos os avanços tecnológicos, a consulta odontológica, por si só, ainda é uma condição estressante para muitas pessoas. O temor ao tratamento odontológico gera um problema cíclico e interfere no limiar da percepção dolorosa, influenciando na qualidade de vida destas pessoas (ACHARYA,
2008; ARMFIELD, 2010).

Portanto, a atenção à saúde bucal de pessoas temerosas continua sendo um grande desafio para os cirurgiões-dentistas. E, provavelmente, por isto, se constitua numa constante preocupação das pesquisas, na área odontológica. Assim, tendo em vista a importância de melhor se compreender as condições relacionadas a este comportamento, em diferentes contextos socioculturais, esta investigação teve como proposição caracterizar um grupo de adultos, em situação não-clínica, quanto à ansiedade ao tratamento odontológico.

\section{Materiais e Método}

O estudo se caracteriza como uma pesquisa descritiva, do tipo transversal, mediante levantamento de dados primários. A população-alvo foi constituída por sujeitos adultos que se encontravam em espaços públicos do perímetro urba-

DOI: https://doi.org/10.25110/arqsaude.v19i3.2015.5546

${ }^{1}$ Acadêmica do Curso de Odontologia da Universidade do Vale do Itajaí; Bolsista de Iniciação Científica. E-mail: mazinha_bc_@hotmail.com

${ }^{2}$ Doutora em Odontopediatria; Integrante do Grupo de Pesquisa Atenção à Saúde Individual e Coletiva em Odontologia da Universidade do Vale do Itajaí. E-mail: silmarchiori@univali.br

${ }^{3}$ Mestre em Educação e Ciências; Integrante do Grupo de Pesquisa Atenção à Saúde Individual e Coletiva em Odontologia da Universidade do Vale do Itajaí. E-mail: erabaldo@univali.br

Endereço de Correspondência: Elisabete Rabaldo Bottan, Rua Uruguai, 458 - Bloco 14, sala 202 - Itajaí/SC - CEP:88302-202, Fone/Fax: 47-33417564 - E-mail: erabaldo@univali.br 
no central da cidade de Balneário Camboriú (Santa Catarina).

A escolha da cidade foi determinada por conveniência, pois, se encontra na área de abrangência da Universidade do Vale do Itajaí, que há vinte anos mantém um curso de graduação em Odontologia.

O plano amostral foi não probabilístico e a obtenção da amostra deu-se por conveniência. Os critérios de inclusão foram os seguintes: a) ter condições físicas e mentais para compreender e responder às questões do instrumento de coleta de dados; b) possuir idade igual ou superior a 18 anos; c) aceitar, por livre e espontânea vontade, participar da pesquisa, assinando o Termo de Consentimento Livre e Esclarecido.

Para a coleta de dados, os sujeitos foram contatados, por um único pesquisador, em espaços públicos. A escolha por estes locais deu-se em função deles reunirem, simultaneamente, diversos grupos de pessoas, que refletem a população em geral. O período destinado à coleta dos dados foi de julho a novembro de 2011.

O instrumento de coleta de dados foi um questionário dividido em três campos. No primeiro campo, constavam três questões para se obter informações sobre gênero, idade e escolaridade. No segundo, foram incluídas duas questões sobre periodicidade e motivos da consulta odontológica. $\mathrm{O}$ terceiro campo se constituiu de uma versão modificada da Dental Anxiety Scale - DAS (CORAH, 1969).

A DAS é uma escala psicométrica que classifica os indivíduos em temerosos, ou não, em relação ao tratamento odontológico. A versão utilizada neste estudo constou de quatro questões, cada uma com quatro alternativas de resposta. Para cada alternativa, é atribuído um valor, em ordem crescente: "a" 1,0 ponto, "b" 2,0 pontos, "c" 3,0 pontos e "d" 4,0 pontos.

O somatório dos escores define o perfil dos sujeitos quanto à ansiedade ao tratamento odontológico, nos seguintes termos: até 04 pontos - não apresenta ansiedade; de 05 a 08 pontos - baixo grau de ansiedade; de 09 a 12 pontos moderado grau de ansiedade; de 13 a 16 pontos - exacerbado grau de ansiedade.

Os dados coletados foram tabulados e organizados com o auxílio de uma ferramenta implementada nas linguagens: HTML (HyperText Markup Language), PHP (Hypertext Preprocessor) e banco de dados MySQL (Structured Query Language). Foi calculada a frequência relativa para cada grau de ansiedade ao tratamento odontológico, segundo o gênero e a faixa etária

Por tratar-se de uma pesquisa envolvendo seres humanos, foram respeitados os preceitos éticos da resolução 466/12. O projeto da pesquisa foi submetido e aprovado pela Comissão de Ética em Pesquisa da UNIVALI, protocolado no SISNEP sob n ${ }^{\circ}$ 0014.0.223.000-05.

\section{Resultados}

O número total de pessoas que integraram a amostra foi de 304 , sendo $56 \%$ do gênero feminino e $44 \%$ do masculino. As idades variaram de 18 a 65 anos. Com relação ao grau de escolaridade, a maioria (69\%) possuía terceiro grau, seguido por $25 \%$ com segundo grau e $6 \%$ com primeiro grau.

Quanto à avaliação da ansiedade ao tratamento odontológico, obteve-se uma pontuação média de 6,3 a qual classifica o grupo como portador de baixo grau de ansiedade. A pontuação média para os homens foi de 5,9 e para as mulheres foi um pouco mais elevada, ou seja, 6,6 pontos.

$\mathrm{Na}$ figura 1, está a frequência de sujeitos para cada grau de ansiedade, onde se pode observar que há um declínio da frequência à medida que o grau se eleva.

Figura 1: Frequência dos graus de ansiedade ao tratamento odontológico da amostra.

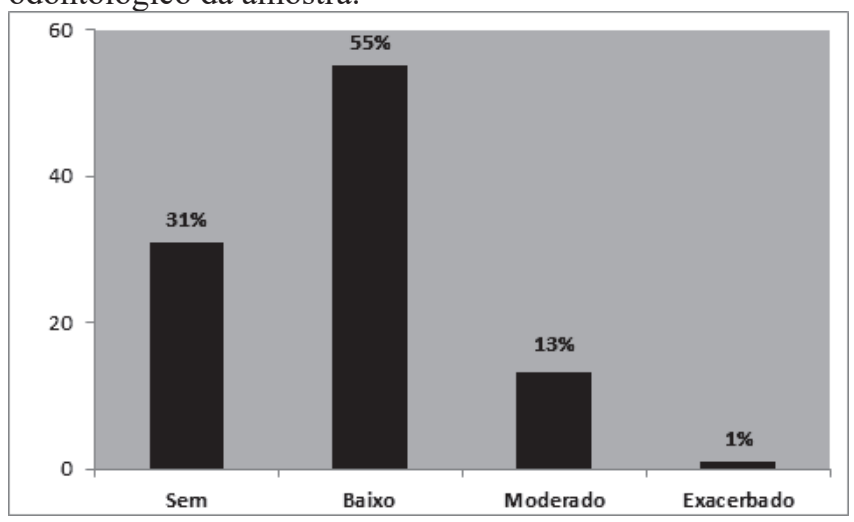

Entre os pesquisados, 208 (69\%) tiveram pontuação igual ou superior a cinco (5), indicando que a maioria é portadora de ansiedade. No grupo dos portadores de ansiedade $(n=208)$ 20,2\% classificaram-se nos graus mais elevados (moderado e exacerbado) e 79,8\% no grau baixo.

A distribuição da frequência relativa de cada grau de ansiedade ao tratamento odontológico, segundo o gênero, consta da figura 2. O percentual de mulheres que manifestaram alguma ansiedade (78\%) foi superior ao dos homens $(58 \%)$. Nos graus moderado e exacerbado, a diferença entre o número de homens e mulheres é reduzida.

Figura 2: Graus de ansiedade ao tratamento odontológico, segundo o gênero.

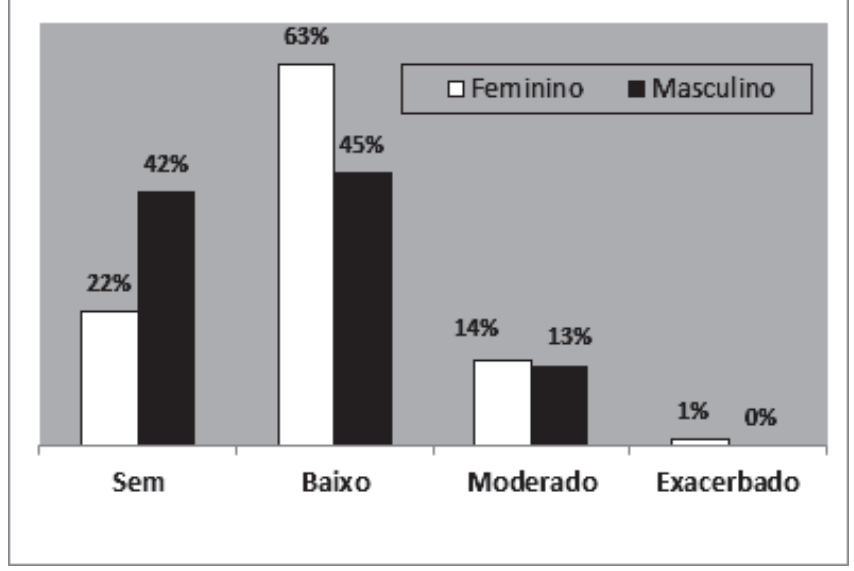

Os dados referentes à relação entre faixa etária e grau de ansiedade estão dispostos na tabela 1, onde se identifica que os níveis sem ansiedade e baixo grau são mais frequentes entre os sujeitos mais jovens. 
Tabela 1: Graus de ansiedade ao tratamento odontológico, segundo a faixa etária.

\begin{tabular}{l|ccccc}
\hline & 18 a 24 anos & 25 a 34 anos & 35 a 44 anos & 45 a 54 anos & 55 ou mais anos \\
\hline Sem & $38,9 \%$ & $23,2 \%$ & $10,5 \%$ & $15,8 \%$ & $11,6 \%$ \\
Baixo & $32,5 \%$ & $25,3 \%$ & $12,7 \%$ & $16,9 \%$ & $12,7 \%$ \\
Moderado & $29,3 \%$ & $29,3 \%$ & $17,1 \%$ & $17,1 \%$ & $7,3 \%$ \\
Exacerbado & $0 \%$ & $0 \%$ & $50 \%$ & $0 \%$ & $50 \%$ \\
\hline
\end{tabular}

Os dados obtidos para cada uma das quatro questões que constituíram a escala de avaliação da ansiedade mostraram que as questões 1 e 2 registraram as maiores frequências para a alternativa sentir-se tranquilo. Na questão 1, que abordava a possibilidade de efetivação de consulta odontológica no dia seguinte, os percentuais foram de $60,8 \%$, entre as mulheres, e 71,4\%, entre os homens. A questão 2, que questionava como a pessoa se sentia ao estar aguardando pelo atendimento na sala de espera, os valores, para a alternativa tranquilo, foram $55 \%$, para as mulheres e $73,7 \%$, para os homens. No entanto, nas questões 3 e 4 , que reportavam sobre a utilização de instrumentos adotados em procedimentos curativos, observou-se uma redução da frequência da alternativa sentir-se tranquilo, principalmente para o gênero feminino $(34,3 \%$, entre as mulheres e $50,9 \%$, entre os homens).

Ao serem questionados sobre com que frequência costumam efetivar a consulta odontológica, a maioria $(75,3 \%)$ afirmou que entre 6 meses e um ano. O maior intervalo de tempo e a consulta em decorrência de dor foram respostas mais frequentes entre os indivíduos do grau exacerbado (Tabela 2). Com relação aos motivos da última consulta odontológica, a maioria $(57,4 \%)$ se referiu a procedimentos curativos e $42,6 \%$ reportaram procedimentos preventivos.

Tabela 2: Periodicidade com que os pesquisados realizam a consulta odontológica.

\begin{tabular}{l|cccc}
\hline \multirow{2}{*}{ Grau de } & \multicolumn{4}{|c}{ PERIODICIDADE DA CONSULTA ODONTOLÓGICA } \\
\cline { 2 - 5 } Ansiedade & 6 meses a 1 ano & A cada 2 anos & Quando tem dor & Não lembro \\
\hline Sem & $71,3 \%$ & $9,6 \%$ & $8,5 \%$ & $10,6 \%$ \\
Baixo & $81,3 \%$ & $4,2 \%$ & $6,0 \%$ & $8,4 \%$ \\
Moderado & $67,5 \%$ & $7,5 \%$ & $15 \%$ & $10 \%$ \\
Exacerbado & $0 \%$ & $25 \%$ & $25 \%$ & $50 \%$ \\
\hline
\end{tabular}

Ao se analisar motivo da consulta em função do grau de ansiedade, identificou-se que a frequência dos motivos preventivos decresce à medida que aumenta o grau de ansiedade (Figura 3).
Figura 3: Motivos da consulta odontológica

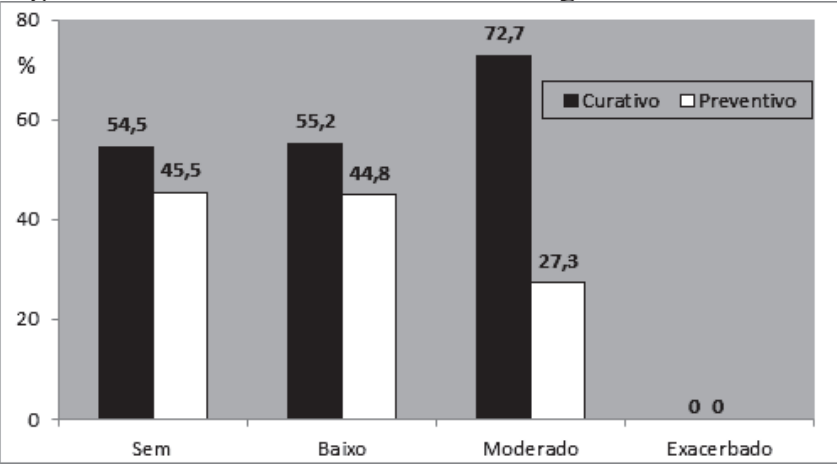

\section{Discussão}

A ansiedade ao tratamento odontológico ainda ocorre em boa parte da população. As manifestações de ansiedade podem ser agrupadas em quatro campos: cognitivo, emocional, comportamental e somático. No campo cognitivo tem-se as situações em que os aspectos não agradáveis relacionados à consulta odontológica, que podem ser apreendidos a partir das mensagens de familiares e/ou amigos ou mediante experiências negativas anteriores, interferem na concentração dos sujeitos, estabelecendo um estado de exacerbada vigilância da atenção. As manifestações no campo emocional geralmente são caracterizadas por crises de choro e apreensão. Quanto ao campo comportamental, as manifestações referem-se a comportamentos não adaptativos, tais como evitar ou fugir da consulta odontológica. E no campo somático as manifestações decorrem da ativação do sistema nervoso autônomo e se apresentam, geralmente como falta de ar, transpiração acentuada e palpitações. Portanto, os sujeitos ansiosos podem apresentar os mais diversos sinais e sintomas, tais como: pensamentos de apreensão, desconforto, inquietação, evitação, palpitação, tensão muscular, tremores, desconforto epigástrico, dentre outros (LIMA ALVAREZ; CASANOVA RIVERO, 2006; ACHARYA, 2008; SHARIF, 2010; RÍOS ERAZO; HERRERA RONDA; ROJAS ALCAYAGA, 2014).

A prevalência deste comportamento tem sido bem estudada em diferentes populações e culturas, nos últimos trinta anos. As pesquisas indicam que a ansiedade tem uma origem multifatorial e é influenciada por fatores sociodemográficos, comportamentais e físicos. Dentre os fatores sociodemográficos, a literatura destaca gênero, idade, nível educacional e econômico. Os fatores comportamentais envolvem aspectos próprios da personalidade de cada indivíduo. Com respeito aos fatores físicos, o mais evidenciado na literatura refere-se às experiências odontológicas traumáticas vivenciadas, em especial, na infância (LIMA ALVAREZ; CASANOVA RIVERO, 2006; RÍOS ERAZO; HERRERA RONDA; ROJAS ALCAYAGA, 2014).

Nem todos os grupos temem ao tratamento odontológico de modo semelhante (ENKLING; MARWINSKI; JÖRHEN, 2006; ÁSTRØM; SKARET; HAUGEJORDEN, 2011; RÍOS ERAZO; HERRERA RONDA; ROJAS ALCAYAGA, 2014). Na literatura revisada, identificou-se que a prevalência de sujeitos que manifestam ansiedade ao tratamento odontológico pode variar de $5 \%$ a $40 \%$ e, de modo geral, a prevalência geral é de 48\% (MANIGLIA-FERREIRA et al., 2004; ENKLING; MARWINSKI; JÖRHEN, 2006; 
LIMA ALVAREZ; CASANOVA RIVERO, 2006; KIROVA et al., 2010; MALVANIA; AJITHKRISHNAN, 2011; CARVALHO et al., 2012). Neste estudo, 69\% dos pesquisados apresentaram algum grau de ansiedade, dos quais $20 \%$ foram classificados nos graus mais elevados, conforme critérios estabelecidos pela escala DAS modificada.

As diferenças nos percentuais apresentados pelos estudos podem ser explicadas em função da metodologia de coleta de dados, pois, embora a maioria dos trabalhos adote a escala de Corah (DAS), em muitos deles, inclusive nesta investigação, este instrumento sofreu modificações. Outros fatores que, também, podem explicar essas diferenças são as variáveis demográficas e as experiências relacionadas ao tratamento odontológico (ARMFIELD, 2010).

A literatura é consistente no que se refere ao fato de que as mulheres são mais ansiosas dos que os homens; situação que, também, se confirmou neste estudo. Esse tipo de comportamento pode ser atribuído a fatores, tais como: as mulheres participam mais de pesquisas do que os homens; as mulheres, em decorrência das normas e condutas sociais, expressam com mais liberdade seus anseios e expectativas; e as mulheres efetivam mais a consulta odontológica dos que os homens (MANIGLIA-FERREIRA et al., 2004; CHAVES et al., 2006; FARAH, 2010; MIALHE et al., 2010; ÁSTRØM; SKARET; HAUGEJORDEN, 2011; GUIOTTI; GONÇALVES; GOULART, 2011 ; CARVALHO et al., 2012).

No que se refere à saúde, os homens, muitas vezes, negam a existência de dor ou sofrimento, de vulnerabilidades, para reforçar a ideia de força, de virilidade do gênero masculino. Assim, os homens têm maior dificuldade de buscar por assistência em saúde em razão de sua autopercepção quanto à necessidade de cuidados e, também, pela noção de que esta é uma tarefa das mulheres (MACHIN et al., 2011).

Quando se analisou a literatura sobre a relação entre idade e ansiedade, foram identificadas duas correntes de pensamento. Alguns autores reportam que os sujeitos idosos, quando comparados aos jovens, são mais temerosos ao tratamento odontológico (MANIGLIA-FERREIRA et al., 2004; CARVALHO et al., 2012). Para outros, ao contrário, os indivíduos jovens são mais temerosos que os idosos (ENKLING; MARWINSKI; JÖRHEN, 2006; FARAH, 2010; ÁSTRØM; SKARET; HAUGEJORDEN, 2011). E os achados desta pesquisa ratificam a primeira corrente, pois entre as faixas etárias mais jovens (18 a 34 anos) é que se encontrava a maior frequência de sujeitos sem ansiedade.

Quanto à influência das experiências relacionadas com o tratamento odontológico, a literatura reforça o conceito de que uma experiência traumática em relação ao tratamento odontológico é a principal razão do aparecimento e do desenvolvimento do medo à consulta odontológica (NICOLAS et al., 2007; BORREANI; WRIGHT; SCAMBLER, 2008; ARMFIELD, 2010; FARAH, 2010; MIALHE et al., 2010; GUIOTTI; GONÇALVES; GOULART, 2011; MALVANIA; AJITHKRISHNAN, 2011). É oportuno, também, destacar a influência do ambiente familiar (no sentido da teoria do modelo) e das histórias relatadas por outras pessoas do convívio social na emergência e desenvolvimento da ansiedade ao tratamento odontológico (LIMA ALVAREZ; CASANOVA RIVERO, 2006; NICOLAS et al., 2007; BORREANI; WRIGHT; SCAMBLER, 2008; ARMFIELD, 2010; FARAH, 2010; MIALHE et al., 2010; GUIOTTI; GONÇAL-
VES; GOULART, 2011; MALVANIA; AJITHKRISHNAN, 2011).

Estudos em diferentes contextos socioculturais evidenciam que as experiências negativas no consultório odontológico geram ansiedade ao tratamento odontológico. Estas experiências negativas podem ser decorrentes de imperícia técnica do profissional ou mesmo por um relacionamento paciente-profissional inadequado. Há, também, determinadas situações da clínica odontológica que são consideradas como desencadeadoras de medo ao tratamento odontológico, tais como odor de produtos farmacológicos, ruído de equipamentos, sangue, instrumentais cirúrgicos, anestesia, dentre ouros. A caneta de alta rotação desperta temor no paciente durante o atendimento odontológico devido ao seu aquecimento sobre a estrutura dentária, gerando uma sintomatologia dolorosa. Além disso, o ruído que ela provoca é frequentemente associado a esta sintomatologia, trazendo sempre a lembrança de dor e desconforto (MASTRANTÔNIO et al., 2010). Além do mais, o fato de o dentista colocar espelhos, sondas, pinças, brocas na boca do paciente provoca, em alguns, o medo de que a língua seja cortada, que a bochecha seja perfurada, entre outras sensações.

Neste sentido, é oportuno lembrar a trajetória da Odontologia. Nos seus primórdios quando praticada por barbeiros e práticos, verdadeiras mutilações eram causadas às pessoas em função de extrações dentárias realizadas com técnicas rudimentares, sem instrumentos adequados e sem higiene. Posteriormente, muito embora com o advento da ciência odontológica, por muitos anos a abordagem cirúrgico-restauradora foi predominante, quando a busca pela atenção odontológica ocorria em situações de dor dentária as quais implicam em tratamentos invasivos e doloridos. Este paradigma favoreceu a manutenção da associação entre Odontologia e medo. Embora nas últimas décadas o paradigma cirúrgico-restaurador venha sendo substituído pelo paradigma da prevenção e os procedimentos odontológicos, em decorrência de equipamentos sofisticados e aprimoramento das técnicas, permitam significativamente a redução do desconforto do paciente, a ansiedade ainda persiste.

Investigações reportam que entre os indivíduos mais ansiosos estão aqueles que não realizam consultas com regularidade e quando a efetivavam o fazem motivados pela dor (MIALHE et al., 2004; CHAVES et al., 2006; SILVA et al., 2009; ARMFIELD, 2010; HALVARI et al., 2010 ; CARVALHO et al., 2012). Este tipo de comportamento, também, foi identificado nesta pesquisa, pois, entre os portadores dos graus mais elevados (moderado e exacerbado), estava a maior frequência dos que procuram pelo atendimento odontológico quando sentem alguma dor.

As pessoas que consideram o tratamento odontológico como sendo gerador de algum desconforto, geralmente, desenvolvem comportamentos de fuga da consulta odontológica, adiando-a para situações limítrofes. Estes pacientes merecem uma atenção especial por parte do cirurgião-dentista. O profissional, nestes casos, deverá conduzir o tratamento do modo mais agradável possível, para a adaptação do paciente, até que a situação se torne conhecida e não acarrete emoções negativas (ACHARYA, 2008; BORREANI; WRIGHT; SCAMBLER, 2008; SILVA et al., 2009; FARAH, 2010; SHARIF, 2010).

A gestão eficiente da ansiedade ao tratamento odon- 
tológico é de suma importância e ela deve consistir de uma abordagem multifacetada. Há diversas opções que podem ser empregadas para o controle da ansiedade. Existem abordagens não farmacológicas (comunicação, gestão do comportamento) e as abordagens farmacológicas, como sedação inalatória, sedação e anestesia geral (SILVA et al., 2009; FARAH, 2010; SHARIF, 2010; COSTA et al., 2011).

Diante do impacto negativo que a ansiedade pode exercer sobre o atendimento odontológico, e na qualidade de vida das pessoas, é necessário que se atue em diferentes dimensões, com o intuito de minimizar os efeitos deste comportamento. Uma frente de atuação deve ser dirigida à população, mediante orientações específicas quanto aos cuidados para com a saúde bucal, dentre os quais a efetivação da consulta odontológica com regularidade, e esclarecimentos sobre as novas tecnologias que podem ser adotadas com o objetivo de reduzir as sensações dolorosas, quando de tratamentos mais invasivos. A outra dimensão volta-se ao cirurgião-dentista, no sentido de fazê-lo perceber que o fenômeno da ansiedade, para muitas pessoas, ainda persiste e que a sua principal conduta deve ser a de investigar os temores de seus pacientes, previamente ao atendimento. E, também, fazê-lo compreender que o estabelecimento de uma relação paciente-profissional focada no diálogo contribui de forma eficaz para a mudança dos comportamentos negativos de seus pacientes.

\section{Conclusão}

Considerando-se que a proposição deste estudo foi de caracterizar um grupo de adultos, em situação não clínica, quanto à ansiedade ao tratamento odontológico, pode-se concluir que:

- a frequência de mulheres e de sujeitos com mais idade portadores de ansiedade foi mais elevada quando comparada à frequência de homens e sujeitos mais jovens;

- sujeitos portadores de graus de ansiedade mais elevados efetivam consultas odontológicas em intervalo de tempo maior do que os sujeitos classificados como não portadores ou com baixo grau de ansiedade;

- a frequência da consulta preventiva foi inversamente proporcional ao grau de ansiedade.

\section{Agradecimentos}

Ao Programa de Iniciação Científica Artigo 170/ Governo do Estado de Santa Catarina, processo $n^{\circ} 1651 / 2011$, pelo financiamento da pesquisa.

\section{Referências}

ACHARYA, S. Factors affecting dental anxiety and beliefs in an Indian population. J. oral rehabil., Oxford, v. 35, n. 4, p. 259-267, 2008.

ARMFIELD, J.M. Towards a better understanding of dental anxiety and fear: cognitions vs experiences. Eur. j. oral sci., Copenhagen, v. 118, n. 3, p. 259-264, 2010.

ÅSTRØM, A.N.; SKARET, E.; HAUGEJORDEN, O.

Dental anxiety and dental attendance among 25-year-olds in
Norway: time trends from 1997 to 2007 . BMC oral health, London, v. 11, n. 10, p. 3-7, 2011.

BORREANI, E.; WRIGHT, D.; SCAMBLER, S. Minimising barriers to dental care in older people. BMC oral health, London, v. 8, n. 7, p. 1-15, 2008.

CARVALHO, R.W.F. et al. Ansiedade frente ao tratamento odontológico: prevalência e fatores preditores em brasileiros. Ciênc. saúde coletiva, Rio de Janeiro, v. 17, n. 7, p. 1915-1922, 2012.

CHAVES, A.M. et al. Estudo epidemiológico da ansiedade dos pacientes ao tratamento odontológico. Rev. odontol. UNESP, Marília, v. 35, n. 4, p. 263-268, 2006.

CORAH, N. L. Development of a dental anxiety scale. J. dent.res., Washington, v. 48, p. 59, 1969.

COSTA, A. M. D. D. et al. Conhecimento dos acadêmicos de odontologia sobre sedação consciente com a utilização de óxido nitroso. Odontol. Clín.-Cient., Recife, v. 10, n. 2, p. 137-141, 2011.

ENKLING, N.; MARWINSKI, G.; JÖRHEN, P. Dental anxiety in a representative sample of residents of a large German city. Clin. oral investig., Berlin, v. 10, n. 1, p. 8491, 2006.

FARAH, G. J. Como controlar a ansiedade do paciente frente a procedimentos odontológicos. Rev. Dental Press Periodontia Implantol., Maringá, v. 4, n. 2, p. 33-34, 2010

GUIOTTI, A. M.; GONÇALVES, H. H. S. B; GOULART, J. C. F. Medo e ansiedade no tratamento odontológico. Rev. ABO Nac., São Paulo, v. XIX, n. 1, supl. 2, p. 212-219, 2011.

HALVARI, A. E. M. et al. Motivation and anxiety for dental treatment: Testing a self-determination theory model of oral self-care behavior and dental clinic attendance. Motiv. emot., New York, v.34, p.15-33, 2010.

KIROVA, D. G. et al. Dental anxiety in adults in Bulgaria. Folia med (Plovdiv), Plovdiv, v. 52, n. 2, p. 49-56, 2010.

LIMA ALVAREZ, M.; CASANOVA RIVERO, Y. Miedo, ansiedad y fobia al tratamiento estomatológico. Rev Hum Med [online], Camaguey, v. 6, n. 1, p. 0-0, 2006.

MACHIN, R. et al. Concepções de gênero, masculinidade e cuidados em saúde: estudo com profissionais de saúde da atenção primária. Ciênc. saúde coletiva, Rio de Janeiro, v. 16, n. 11, p. 4503-4512, 2011.

MALVANIA, E. A.; AJITHKRISHNAN, C. G. Prevalence and socio-demographic correlates of dental anxiety among a group of adult patients attending a dental institution in Vadodara city, Gujarat, India. Indian j. dent. Res. [online], Ahmedabad, v. 22, n. 1, p. 179-180, 2011. 
MANIGLIA-FERREIRA, C. et al. Ansiedade odontológica: nível, prevalência e comportamento. RBPS, Fortaleza, v. 17, n. 2, p. 51-55, 2004.

MASTRANTONIO, S. D. S. et al. Redução do medo durante o tratamento odontológico utilizando pontas ultrassônicas. RGO (Porto Alegre), Porto Alegre, n. 1, p. 119-122, 2010.

MIALHE, F. L. et al. Medo odontológico entre pacientes atendidos em um serviço de urgência. Pesq Bras

Odontoped Clin Integr, João Pessoa, v. 10, n. 3, p. 483487, 2010.

NICOLAS, E. et al. A national cross-sectional survey of dental anxiety in the French adult population. BMC oral health, London, v. 7, art. 12, 2007. Disponível em: $<$ http:// www.biomedcentral.com/1472-6831/7/12>. Acesso em: 10 dez. 2010.

RÍOS ERAZO, M.; HERRERA RONDA, A.; ROJAS ALCAYAGA, G. Ansiedad dental: Evaluación y tratamiento. Av. Odontoestomatol, Madrid, v. 30, n. 1, p. 39-46, 2014.

SHARIF, M. O. Dental anxiety: detection and management. J. Appl. Oral Sci. [on line], Bauru, v. 18, n. 2, p. i-i, 2010.

SILVA, A. M. et al. Study of emotional and psychological factors that may interfere in the dental treatment. J. Health Sci. Inst., São Paulo, v. 27, n. 3, p. 249-253, 2009. 